\title{
A flume experiment on the effect of constriction shape on the formation of forced pools
}

\author{
D. M. Thompson ${ }^{1}$ and C. R. McCarrick ${ }^{2}$ \\ ${ }^{1}$ Department of Physics, Astronomy and Geophysics, Connecticut College, Campus Box 5585, 270 Mohegan Avenue, New \\ London, Connecticut, 06320, USA \\ ${ }^{2}$ Excel Environmental Resources, Inc., 111 North Center Drive, North Brunswick, NJ 08902, USA
}

Received: 24 February 2010 - Published in Hydrol. Earth Syst. Sci. Discuss.: 22 March 2010

Revised: 28 June 2010 - Accepted: 9 July 2010 - Published: 20 July 2010

\begin{abstract}
A series of 18 flume runs were conducted in a 6-m long, $0.5-\mathrm{m}$ wide recirculating flume with a bed gradient of $0.8 \%$ to determine the influence of obstruction shape on the formation and characteristics of forced pools. Six differentshaped obstructions were added to the flume with the maximum width of the obstruction held constant at $20 \mathrm{~cm}$, which equaled a $40 \%$ constriction of flow. The obstruction shapes used included a square, a rectangle, a right triangle with the hypotenuse-facing upstream, a right triangle with the hypotenuse-facing downstream, a combination of a square and triangle with the hypotenuse-facing upstream, and a rectangle and semi-circle shape. Three flume runs were conducted with each obstruction shape. A profile of the flume bed was taken after each experiment and a grid measurement of bed elevations for the last run were conducted to create topographic maps of the flume bed to compare pool-riffle morphologies. ANOVA results indicate pool depth, pool location, and the distance between the pool center and the riffle crest all vary with the obstruction shape. Obstructions with a more blunt upstream face created deeper pools, more total scour and longer pool-riffle sequence lengths than pools formed by obstructions with a more gradual narrowing of flow. The increased volume of scour associated with obstructions that rapidly narrow flow also creates larger volume riffles that cover a greater extent of the channel bed.
\end{abstract}

\section{Correspondence to: D. M. Thompson} (dmtho@conncoll.edu)

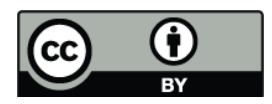

\section{Introduction}

Pool-riffle formation and maintenance is an important process in the establishment of channel morphology and the associated aquatic habitat in rivers. One particular type of pool is termed a "force pool" and is used to describe streambed depressions associated with a physical obstruction to flow (Montgomery et al., 1995). The obstruction creates backwater conditions in the upstream portion of the pool that leads to steep water-surface slopes and flow acceleration past the obstruction (Kieffer, 1985; Schmidt et al., 1993; Thompson et al., 1999). Flow convergence produces jet flow and high velocities in the pool (Kieffer, 1985; Miller, 1994; Wohl and Thompson, 2000), and promotes flow recirculation with associated turbulence generation (Clifford, 1993; Schmidt et al., 1993; Thompson, 2004). These hydraulic patterns help form and maintain the pool morphology and directly link the location of forced pools to the location of obstructions (Thompson, 2001). Therefore, the characteristics of the channel obstruction should directly influence the characteristics of the resultant pool-riffle sequence. A set of flume experiments was conducted to determine the influence of obstruction shape on pool-riffle characteristics. It is hypothesized that pool depth, pool length and pool-riffle sequence length will vary with different-shaped obstructions to flow even if the degree of the constriction is kept constant.

(dmtho@conncoll.edu)

Published by Copernicus Publications on behalf of the European Geosciences Union. 


\subsection{Formation of forced pools and riffles}

Several researchers suggest that the pool-riffle unit probably represents a single morphologic feature linked by sediment and flow dynamics (Clifford, 1993; Thompson, 2001). The pool-riffle sequence itself is comprised of two distinct features. A pool is generally classified as a streambed depression that occupies the main portion of a channel and has a nearly horizontal water-surface slope at low flow (Lisle and Hilton, 1992). A subset of pools formed by obstructions to flow is called forced pools and the remaining pools are termed free-formed pools (Montgomery et al., 1995). Riffles are often identified as areas with steeper water-surface slopes and faster velocities at low flow.

Channel obstructions generate flow convergence and subsequent forced pools (Lisle, 1986; Montgomery et al., 1995; Thompson et al., 1999; Buffington et al., 2002; MacWilliams et al., 2006). In channels with forced pools, over $80 \%$ of pools are associated with structural controls and large obstructions that include boulders, bedrock outcrops and large woody debris (Dolan et al., 1978; Lisle, 1986; Montgomery et al., 1995; Thompson, 2001). For example, Buffington et al. (2002) list obstructions as the primary control on pool formation in 32 Pacific Northwest study reaches.

\subsection{Hydraulics and turbulent patterns in forced pools}

Channel constrictions produce backwater and a local increase in water-surface slope (Kieffer, 1985; Lisle, 1986; Schmidt et al., 1993; Miller, 1994; Thompson et al., 1999). Subsequent development of flow convergence, jet flow, flow separation and recirculation at the constriction create high velocities in the center of the pool (Kieffer, 1985; Lisle, 1986; Schmidt et al., 1993; Thompson et al., 1998; MacWilliams et al., 2006). Thompson et al. (1998) showed that when flow converges in a pool and is forced around the obstruction, it can continue to converge past the physical obstruction to flow and form an even narrower zone of high-velocity flow in the pool center called a vena contracta (Fig. 1). The vena contracta is part of the jet flow and can display a narrower cross-section of downstream flow than at the channel constriction (Thompson et al., 1998). Adjacent to the vena contracta is a large area of recirculating flow in the wake of the constriction. In this area, flows are stagnant in the middle of the recirculating zone and maximum upstream velocities occur adjacent to the channel walls (Thompson et al., 1998). The vena contracta and recirculating eddy are marked by a local minimum in water-surface elevations (Thompson et al., 1998). Immediately downstream of this surface elevation minimum, there is a streamwise increase in water-surface elevation, which creates an adverse pressure gradient (Thompson et al., 1999). In this area, pressure forces work in opposition to the downstream momentum of the flow, resulting in both deceleration and strong lateral spreading of the downstream flow (Thompson et al., 1998).

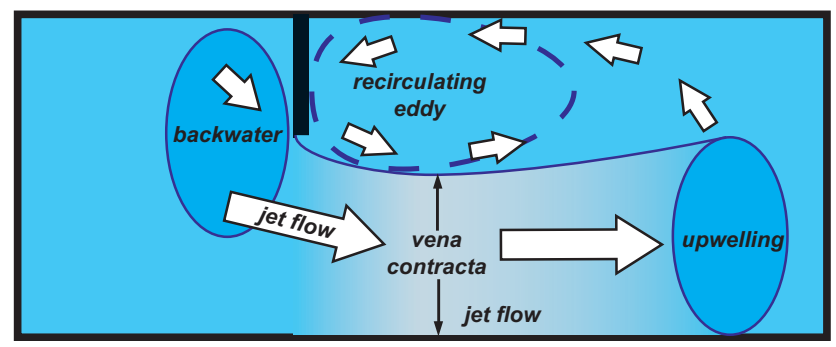

Fig. 1. Plan view of conceptual model of vena-contracta formation.

Researchers report that vortex shedding from vertical shear layers is a common feature in forced pools that may create scour (Lisle, 1986; Clifford, 1993; MacVicar and Roy, 2007a; Thompson, 2007). Turbulence plays an important role in pool scour because it creates instantaneous forces greater than time-averaged values (Nelson et al., 1993; McLean et al., 1994; De Serres et al., 1999; Roy et al., 1999). As a result, sediment particles in pools can be detached and entrained by turbulent bursts and fluctuations even when mean shear stress is less than the time-average critical shear stress for that material because the instantaneous differences of pressure on the upper and lower surfaces of large bed material generate transient uplift forces (Liu et al., 1998; Jia et al., 2001; Hassan and Woodsmith, 2004). The differences in turbulence intensities and sediment characteristics between pools and riffles help to maintain disparities in sediment movement along the sequence (Clifford, 1993).

Strong turbulence fluctuations are associated with vertical separation zones or shear layers that originate when a boundary layer separates from a lateral flow constriction (Kieffer, 1985; Clifford, 1993; Thompson, 2006; MacVicar and Roy, 2007b). Turbulence generation and decay in pools is strongly impacted by areas of flow acceleration and deceleration especially in areas of rapid changes in depth or width associated with flow convergence (Wohl and Thompson, 2000; MacVicar and Roy, 2007b). Obstacles create wakes with shedding of quasi-periodic vortex structures that fixed the location of scour and deposition (Clifford, 1993; Thompson, 2007). Vortices along the shear layer represent intense turbulence capable of removing smaller particles from the bed of the pool (Kieffer, 1985; Harrison and Keller, 2007; MacVicar and Roy, 2007a; Thompson, 2007). Downstream of the constriction, lateral flow divergence, upwelling and boil formation along the pool-exit slope encourage deposition at the tail of the pool and head of the riffle (Petit, 1987; Sear, 1996; Thompson et al., 1996; Milan et al., 2001; Thompson and Wohl, 2009). 


\subsection{The influence of channel constrictions on pool-riffle characteristics}

Approach conditions influence pool size and shape because they impact the strength of flow convergence and jet flow. In forced pools, a narrower constriction ponds more water upstream of the obstruction to flow and increases the water-surface gradient entering the pool (Kieffer, 1985), produces stronger jet flow (Kieffer, 1989), and scours deeper and longer pools (Lisle and Hilton, 1992). Based on conservation of mass, more severe restrictions create higher velocities under steady flow conditions (Kieffer, 1985). Several field studies show the connection between degree of constrictions and pool volume (Kieffer, 1985; Thompson and Hoffman, 2001; Buffington et al., 2002; Wohl and Legleiter, 2003). Lisle (1986) even estimated the minimum obstruction size needed to create a pool.

The shape and orientation of constrictions also influences pool characteristics. Buffington et al. (2002) found that the largest, best developed pools were created by vertical versus horizontal constrictions. Lisle (1986) found that deflection angle of the flow at an obstruction influenced pool development. Thompson (2002) found that taller and narrower constrictions in a flume created deeper pools than wider lower features with the same degree of constriction because of differences in backwater development. In a different flume experiment, Thompson (2006) isolated the influence of vortex shedding from lateral constrictions by comparing a streamlined and non-streamlined obstruction to flow, and showed that pools were deeper and shorter with the nonstreamlined obstructions presumably due to enhanced vortex shedding along vertically-oriented shear zones downstream of the obstruction. In a field study, Thompson and Hoffman (2001) found that higher gradients and wider approach sections, and narrower constrictions tend to be associated with deeper pools. Meanwhile, longer pools tend to be associated with longer channel constrictions and increased downstream distance to the constrictions (Thompson and Hoffman, 2001). Similarly, Wohl and Legleiter (2003) found deeper and longer pools with narrower constrictions, greater cumulative upstream drops in elevation and steeper approach gradients.

The existing literature show that scour of a forced pool results from a complex set of interactions associated with flow convergence, jet flow, wake zones and vortex shedding. The main objective of this study was to determine how sensitive pool-riffle characteristics were to changes in the shape of obstructions that produced the same constriction ratio. Because different obstruction shapes should influence flow convergence and the degree of vena-contracta narrowing. Constrictions that create better developed vena-contracta with narrow jets of high velocity should produce more pool scour. Increased scour should produce deeper and longer pools with better developed riffles downstream of the pool.

\section{Methods}

Six sets of unscaled flume experiments were conducted in a 6-m long, 0.5-m wide recirculating flume with a channel-bed gradient of $0.8 \%$. Isotropic turbulence conditions were created at the head of the flume through the utilization of a gridded baffle consisting of 6-mm circular openings. The bed of the flume was filled with uniform gravel with a $d_{16}, d_{50}$ and $d_{84}$ of $4.1 \mathrm{~mm}, 5.4 \mathrm{~mm}$ and $7.0 \mathrm{~mm}$, respectively. During the experiments sediment transport upstream of the constrictions was limited because of backwater development from the obstruction. Sediment that exited the downstream of the flume was captured in a sediment trap and was not recirculated. The bed gravel was smoothed before each experiment to ensure similar initial conditions for pool formation. The flume was slowly filled with water to limit scour from the initial rush of flow across the bed. Initial water-surface elevations were kept constant for all flume runs. Discharge was then rapidly increased and held constant at $20 \mathrm{l} / \mathrm{s}$ for a total duration of $30 \mathrm{~min}$ for each flume run. Trial runs with longer durations confirmed little or no change in flume-bed elevations with longer duration experiments. Therefore, the surveyed pool-riffle topography are assumed to represent equilibrium dimensions.

In each set of flume experiments, a different-shaped obstruction was centered $300-\mathrm{cm}$ downstream from the head of the flume. The obstructions were uniform in the vertical direction and high enough to prevent overtopping by all flows. The maximum widths of the obstructions were held constant at $20 \mathrm{~cm}$ for each shape to create the same degree of constriction with a resulting constriction ratio of $40 \%$. Six shapes were used in a crude attempt to mimic the shapes most found in nature (Fig. 2). In plan view, these obstruction shapes included a square, a rectangular, a trapezoid hybrid of a right triangle and a square (trapezoid), a composite cylindrical and rectangular object (semi-circle), and a right triangle with the hypotenuse-facing both upstream (triangle UP) and downstream (triangle DS). The resultant objects created a variety of different longitudinal lengths, and upstream and downstream angles to flow. Natural obstructions are more complex, but contain many of the basic elements depicted with these simplified shapes. Care was taken to assure that each obstruction was placed flush on the floor and left wall of the flume to make certain all discharge passed through the single opening on the right side of the flume.

Each set of six flume experiments consisted of three flume runs with the same obstruction shape. After each run, a longitudinal profile was taken along the flume center line, which was positioned $5-\mathrm{cm}$ from the outer edge of the obstructions. Flume-bed elevations were measured with a digital point gauge at $5-\mathrm{cm}$ intervals from $250-\mathrm{cm}$ to $380-\mathrm{cm}$ downstream of the head of the flume. In addition to the centerline longitudinal profile, a grid of the flume bed was taken on the third run of each set of experiments. Measurements were collected at $5,15,25,35$, and $45 \mathrm{~cm}$ from the right wall of the 


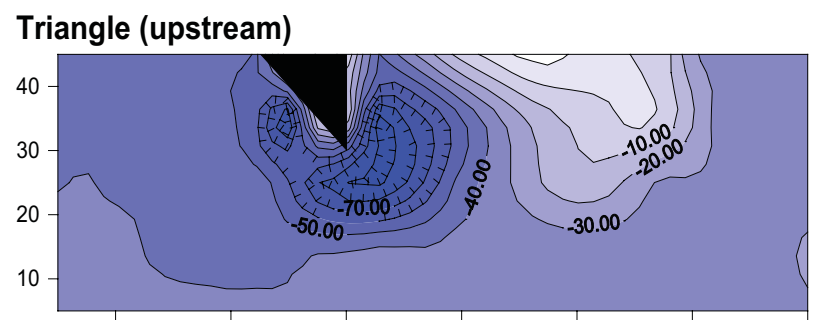

Trapezoid

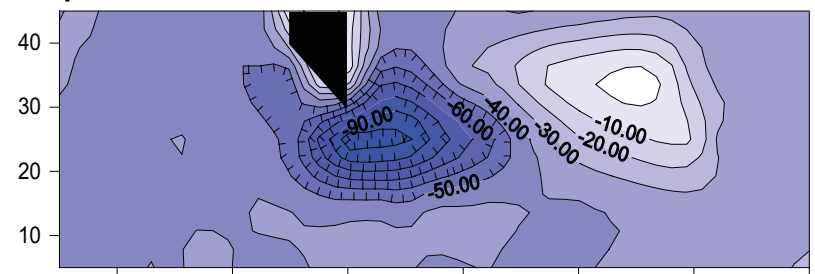

\section{Semi-circle}

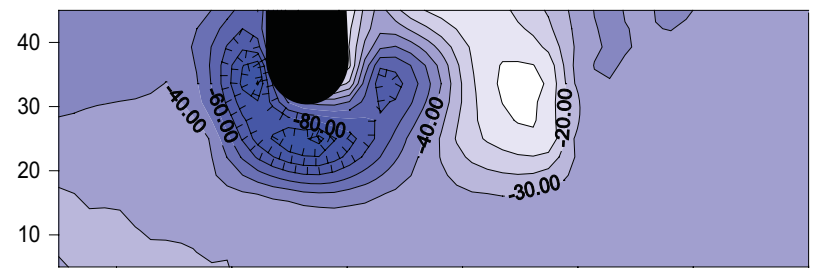

Triangle (downstream)

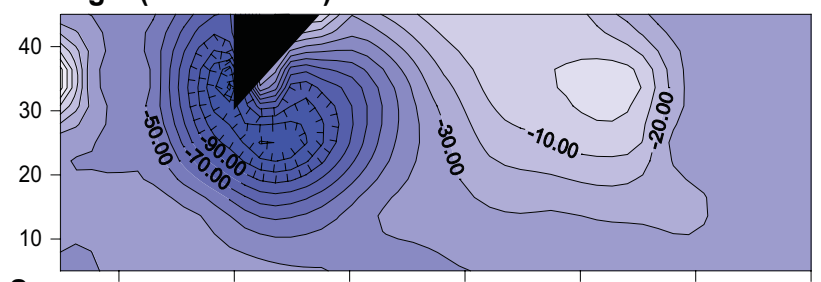

Square

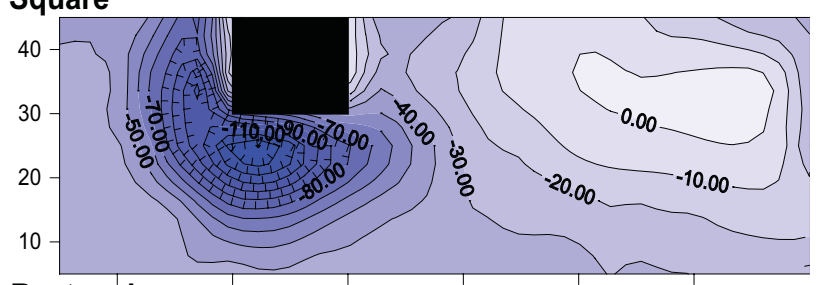

Rectangle

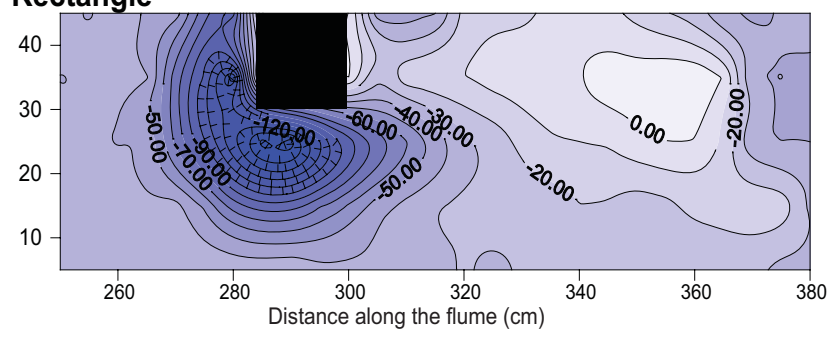

Fig. 2. Plan view of flume-bed topography for pool-riffle sequences formed by different shaped obstructions to flow.

flume along the same $130 \mathrm{~cm}$ length of flume bed used for the longitudinal profile. These measurements were used to create topographic maps of the flume bed.
The bed elevation and location of the pool center and riffle crest were used as indicators of pool shape. For this analysis the pool center was defined as the local elevation minima and the riffle crest as the local maxima along the flume centerline within the measurement area. Maximum scour depths usually occurred along the flume centerline, so centerline profiles provide a good measure of pool characteristics. However, the lateral position of the riffle crest varied with obstruction shape which complicates the analyses of those results. A fifth variable for the pool-riffle sequence length was computed as the distance between the minimum and maximum elevations. These five response variables were used in a series of one-way analysis of variance (ANOVA) and TukeyKramer HSD comparison of means statistical tests to determine if obstruction shape influenced pool and riffle characteristics.

Because the obstruction shape seemed to dominate the overall characteristics of the length of the full pool-riffle sequence, a second set of ANOVA tests were performed to better understand the control of obstruction shape on poolcenter location. In particular, the distances from the upper edge, middle and lower edge of the point of maximum constriction to the pool and riffle were analyzed. In the case of pointed and the semi-circle shaped obstructions, the point of maximum constriction is a point and the three different distances were equivalent. However, the two blunt obstructions represented by the square and rectangle create maximum degrees of constriction along their entire length. As a result, the distance from the point of maximum constriction varies when measured from the upper edge, middle and lower edge of the obstruction. In this analysis, an effort was conducted to determine if one of the various measures of distance from the constriction yielded a result of no significant differences for any of the obstruction shapes. The thought was that eliminating differences in response variable would highlight the fundamental control on what determines the total distance from the obstruction to the pool center. Once the analysis was completed, the downstream distance to the riffle crest was reanalyzed with ANOVA and a comparison of means test utilizing the new definition for the position of the obstruction.

\section{Results}

Observations during the flume runs confirm that flow patterns were generally consistent for each series of three runs, but varied somewhat for each set of experiments when obstruction shapes were altered. Each obstruction created backwater with associated flow convergence and jet flow that subsequently formed both a pool and riffle. The pool began to form almost the instant the flow rate was increased to $201 / \mathrm{s}$. Scour appeared to begin at the first point of greatest restriction, but the location of maximum scour gradually progressed downstream. Material removed in the scouring 
Table 1. Volumes of pool scour and sediment deposited as riffle for the different obstructions.

\begin{tabular}{lrrrr}
\hline Obstruction shape & $\begin{array}{r}\text { Pool } \\
\text { volume }\left(\mathrm{cm}^{3}\right)\end{array}$ & $\begin{array}{r}\text { Pool bed } \\
\text { area }\left(\mathrm{cm}^{2}\right)\end{array}$ & $\begin{array}{r}\text { Riffle sediment } \\
\text { volume }\left(\mathrm{cm}^{3}\right)\end{array}$ & $\begin{array}{r}\text { Riffle bed } \\
\text { area }\left(\mathrm{cm}^{2}\right)\end{array}$ \\
\hline Trapezoid & 3144 & 2599 & 1444 & 965 \\
Triangle (UP) & 3981 & 3206 & 1831 & 1045 \\
Semi-circle & 4074 & 3907 & 1540 & 1017 \\
Triangle (DS) & 4790 & 3459 & 2422 & 1496 \\
Rectangle & 6987 & 3722 & 4043 & 2520 \\
Square & 8028 & 3761 & 4858 & 2674 \\
\hline
\end{tabular}

process was transported downstream and deposited. The pool-riffle formation process slowed noticeably to negligible levels during the later third of the experiment.

Differences in the location of the pool and riffle in relation to the obstruction were obvious at the conclusion of many experiments. Some pools were located off the back edge of the obstruction, while other pools formed either off the front edge or in the center of the obstruction (Fig. 2). There was variation in the width of the pools that appears to correlate with differences in the depth of scour. However, the position of maximum scour appears to occur along the flume centerline for all obstruction shapes. Riffles were formed at locations varying in distance downstream, but also in locations from the right to the left wall of the flume.

The longitudinal plots of flume-bed elevation show minor differences in the location of the pool center even when adjusted for the distance from the upstream edge of the constriction (Fig. 3). The longitudinal profiles also indicate large differences in the depth of pools for the six different shaped obstructions. The deepest pools were associated with obstructions with blunt upstream edges that include the square, rectangle and triangle DS. Pools became progressively shallower when the front edge of the obstruction was angled more in the downstream direction as was the case for the trapezoid, semi-circle and triangle UP.

Differences in the height of the riffle crest appear fairly small on the longitudinal profile (Fig. 3). However, it should be noted that the riffle crest did not always occur in the same lateral position, which would influence the profile shape along the flume centerline. Although water-surface elevations were not measured, observations during experiments suggest that riffle-crest heights began to approach the water surface, which may have acted as a limit of riffle-crest elevation. However, the volume of sediment deposited in the riffle varied with obstruction shape (Table 1). In particular, obstructions that scoured deep pools tended to deposit this sediment as a larger riffle downstream of the pool. Increased volumes of sediment tended to result in elongation of the mound of material along the riffle instead of producing a higher deposit (Fig. 2).

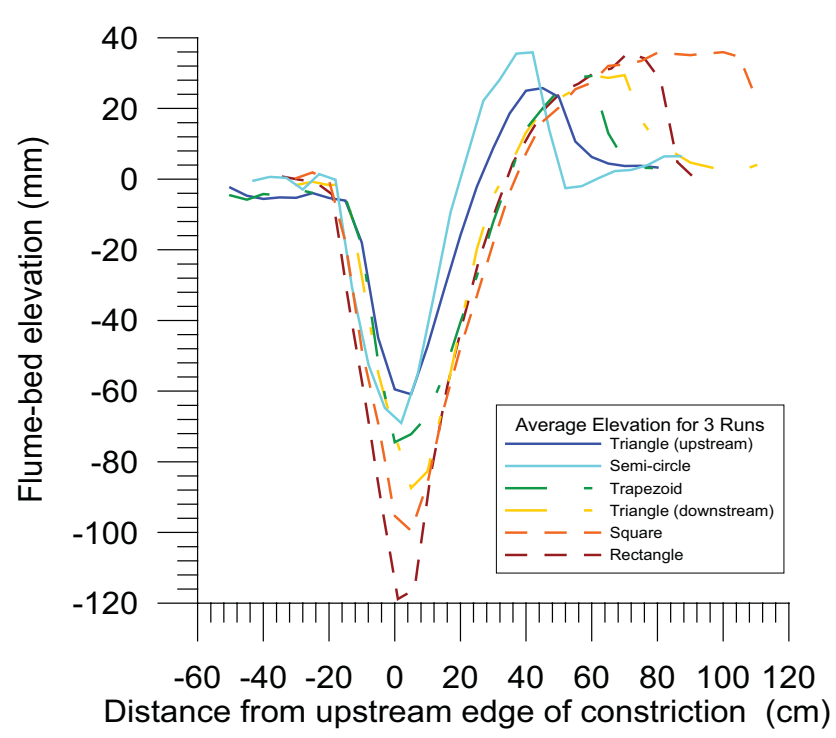

Fig. 3. Longitudinal profiles of flume centerline for pool-riffle sequences formed by different shaped obstructions to flow.

Results for the ANOVA tests with control variables for both obstructions shape and flume run number versus the five various response variables show a simpler statistical model can be used.

In all five analyses for the different pool and riffle characteristics the control variable for obstruction shape but not run number was significant at the $\alpha=0.05$ level. P-values for the obstruction shape variable varied from less than 0.0001 to a maximum of 0.0240 . Conversely, p-values for flume run number varied from 0.1045 to 0.8022 . Therefore, the set of three runs for each obstruction shape appear to be repeatable and the data from all experiments was combined and analyzed as a single control variable for obstruction shape with analysis of variance and comparison of means tests.

The characteristics of the pool were generally more responsive to changes in obstruction shape than were the characteristics of the riffle. The ANOVA test for obstruction shape versus maximum pool depth was significant with a p-value of less than 0.0001 and an $R^{2}$ of 0.977 (Table 2). The comparison of means indicate the rectangle, square and 
Table 2. Analysis of variance results for obstruction shape versus the five response variables.

\begin{tabular}{lrc}
\hline Response variable & p-value & $R^{2}$ \\
\hline Pool depth & $<0.0001$ & 0.977 \\
Riffle crest height & 0.0022 & 0.756 \\
Location of pool center & $<0.0001$ & 0.955 \\
Location of riffle crest & 0.0426 & 0.577 \\
Pool center to riffle crest distance & $<0.0001$ & 0.884 \\
\hline
\end{tabular}

triangle DS all had a unique influence on pool depth with maximum depth associated with the rectangle (Table 3). Furthermore, the triangle UP was significantly different than the trapezoid shape. However, there were no significant differences between the semi-circle and the trapezoid or triangle UP because depths for the semi-circle tended to be intermediate and overlap the other two shapes. The magnitude of difference was also on the decimeter scale. In comparison, riffle crest height was significantly related to obstruction shape with a p-value of 0.0022 and an $R^{2}$ of 0.756 . Fewer differences in the height of riffle crest exist among the six obstruction shapes and those differences were only at the centimeter scale. The triangle UP differed from the rectangle, semicircle and square, but no other obstruction shapes showed significant differences.

The data on location of pool centers and riffle crests showed similar trends. The ANOVA test for obstruction shape versus location of the pool center was significant with a p-value of less than 0.0001 and an $R^{2}$ of 0.955 (Table 2). The comparison of means indicate three distinct groups. The square, rectangle, and triangle DS form a discrete group, the semi-circle produced an unique influence, and the trapezoid and triangle UP appear to group together (Table 3). In contrast, obstruction shape did not create significant differences in riffle crest location based on the more conservative comparison of means test. The ANOVA results for distance from pool center to riffle crest was significant with a p-value of less than 0.0001 and an $R^{2}$ of 0.884 . The comparison of means data suggest a series of groups with more compact pool-riffle morphologies created by semi-circle obstructions and more elongated pool-riffle units with square obstructions.

The second series of ANOVA tests designed to investigate controls on pool center location indicated no significant differences in any mean values for distance from the constriction when the length was measured from the upstream end of the beginning of maximum constriction width to the pool center. The p-value for the ANOVA was 0.2561 with an $R^{2}$ value of 0.387 (Table 4 ). The p-values for distance measured from the mid-point of the constriction and downstream end of the constriction were 0.0001 and less than 0.0001 . These two tests and the comparison of means results indicate differences in location still exist due to obstruction shape when distance from the constriction is defined in these manners
(Table 5). In particular, the square- and rectangular-shaped obstructions appear as distinct from the other four obstruction shapes unless the distance from the constriction is measured from the upstream edge.

An ANOVA and comparison of means test was also computed for the distance from the beginning of maximum constriction width to the riffle crest. The p-value for the ANOVA was 0.0047 with an $R^{2}$ value of 0.720 . The comparison of means indicate some of the differences in the distance from the constriction to riffle crest among the square, rectangle, the semi-circle and triangle UP appearing distinct (Table 6). The trapezoid and triangle DS obstructions had intermediate mean values and were not statistically distinct from any of the other shapes.

\section{Discussion}

The longitudinal profiles, topographic maps of the flume-bed and the statistical results all confirm that forced-pool depth is heavily influenced by constriction shape. Furthermore, pool-riffle sequence length varied with different shaped obstructions. Pools scoured from an initially plane bed tended to be deeper and located further upstream with obstructions that presented a blunt upstream face to the flow. Obstructions with a more gradual transition from the full flume width to the $40 \%$ constriction to flow tended to delay the onset of scour to locations further downstream, and limit the total depth of scour. The results from the 18 flume runs highlight variations in these process that result from slight manipulations in the boundary conditions with the same $40 \%$ restriction to flow.

\subsection{Pool-riffle characteristics versus obstruction shape}

As hypothesized, pool depth varied with obstruction shape (Table 2). The results are highly significant and obstruction shape explains almost all the variation among the 18 flume runs. The actual variation in depth was also relatively large, with some pools over twice as deep as the shallowest pools. The deeper pools tended to be larger in overall extent (Table 1). For the three obstructions with the blunt upstream face, the triangle DS, the square and the rectangle, some scour extended across the entire width of flow in the vicinity of the obstruction (Fig. 2). The other three shapes showed scour isolated within regions closer to the obstruction with no obvious changes in flume-bed elevation closer to the right wall of the flume.

The location of the pool center also varied with obstruction shape (Tables 2 and 3). However, further statistical analysis suggests the differences in downstream distance from the obstruction disappear when the distance is measured from the upstream edge of the first point of maximum constriction (Table 4). These results suggest that the position of the upstream edge of the constriction has a dominate control on the 
Table 3. Comparison of means results for the five response variables. Mean values contained within the same horizontal line are not significantly different from each other. Values in parentheses are the corresponding standard deviations.

\begin{tabular}{|c|c|c|c|c|c|}
\hline \multicolumn{6}{|c|}{ Mean pool depth (mm) } \\
\hline Triangle (UP) & Semi-circle & Trapezoid & Triangle (DS) & Square & Rectangle \\
\hline \multirow[t]{4}{*}{$61(3.7)$} & $69(3.4)$ & & \multirow{4}{*}{$87.4(1.9)$} & \multirow{4}{*}{$99.5(6.0)$} & \\
\hline & $69(3.4)$ & $75.2(3.4)$ & & & \\
\hline & & & & & \\
\hline & & & & & $119.3(2.5)$ \\
\hline \multicolumn{6}{|c|}{ Mean riffle crest height (mm) } \\
\hline Triangle (UP) & Triangle (DS) & Trapezoid & Rectangle & Semi-circle & Square \\
\hline \multirow[t]{2}{*}{$27.6(2.2)$} & $31.6(0.4)$ & $31.7(3.5)$ & & & \\
\hline & $31.6(0.4)$ & $31.7(3.5)$ & $36.2(3.9)$ & $36.5(1.1)$ & $37.4(1.1)$ \\
\hline \multicolumn{6}{|c|}{ Mean location of pool center $(\mathrm{cm})$} \\
\hline Square & Triangle (DS) & Rectangle & Semi-circle & Trapezoid & Triangle (US) \\
\hline \multirow[t]{2}{*}{$285(0.0)$} & $285(0.0)$ & $286.7(2.9)$ & \multirow{2}{*}{$295(0.0)$} & & \\
\hline & & & & $301.7(2.9)$ & $303.3(2.9)$ \\
\hline \multicolumn{6}{|c|}{ Mean location of riffle crest $(\mathrm{cm})$} \\
\hline Semi-circle & Triangle (DS) & Triangle (UP) & Square & Rectangle & Trapezoid \\
\hline $331.7(2.9)$ & $343.3(20.8)$ & $346.7(5.8)$ & $358.3(12.6)$ & $358.3(5.8)$ & $360(5.0)$ \\
\hline \multicolumn{6}{|c|}{ Mean pool center to riffle crest distance $(\mathrm{cm})$} \\
\hline Semi-circle & Triangle (UP) & Trapezoid & Triangle (DS) & Rectangle & Square \\
\hline \multirow[t]{4}{*}{36.7 (2.9) } & $43.3(7.6)$ & & & & \\
\hline & 43.3 (7.6) & $58.3(2.9)$ & & & \\
\hline & & $58.3(2.9)$ & $66.7(7.6)$ & $71.7(7.6)$ & \\
\hline & & & $66.7(7.6)$ & $71.7(7.6)$ & $76.7(7.6)$ \\
\hline
\end{tabular}

Table 4. Analysis of variance results for obstruction shape versus distance from constriction to the pool center. The distance from the maximum width of the constriction is defined differently for each analysis.

\begin{tabular}{lrr}
\hline Control variable & $\mathrm{p}$-value & $R^{2}$ \\
\hline Distance from upstream edge & 0.2561 & 0.387 \\
Distance from mid-point & 0.0001 & 0.852 \\
Distance from downstream edge & $<0.0001$ & 0.960 \\
\hline
\end{tabular}

downstream distance to the pool center. Longitudinal profiles measured in relation to the upper edge of the obstruction show very similar initial locations of scour upstream of the different obstructions (Fig. 3). Furthermore, the final upstream and downstream sediment slopes were similar in almost all cases. Only the semi-circular obstruction seems to create a markedly steeper pool-exit slope. The remaining pools appear roughly symmetrical with regard to upstream and downstream angles of sediment, with similar flume-bed slopes among the different flume runs in the entrance and exit regions of the pool.

The maximum height of the riffle crest showed much less reliance upon shape and much less overall variation (Table 2). However, larger riffle deposits associated with the larger pools (Table 1). It is logical that deep pools with a larger total volume of scour would also produce large deposits of material downstream of the pool. These deposits appear to form distinct riffles instead of depositing more diffusely across the flume bed. Despite some of the differences in riffle shape, it is safe to conclude obstruction-shape had a much more important influence on forced-pool characteristics than the associated riffles.

The downstream location of the riffle crests showed no variation with obstruction shape (Table 2). However, this result was due partly to the way that the position of the constriction was defined. Figure 3 shows that the riffle crest for the semi-circle and triangle UP were positioned further upstream than the remainder of the rifle crests. Similarly, 
Table 5. Comparison of means results for obstruction shape versus distance from constriction to the pool center. The distance from the maximum width of the constriction is defined differently for each analysis. Mean values contained within the same horizontal line are not significantly different from each other. Values in parentheses are the corresponding standard deviations.

\begin{tabular}{crrrrr}
\hline \multicolumn{2}{c}{ Distance from upstream edge $(\mathrm{cm})$} \\
\multicolumn{1}{c}{ Trapezoid } & Semi-circle & Rectangle & Triangle (UP) & Triangle (DS) & Square \\
\hline $1.7(2.9)$ & $2.0(0.0)$ & $2.7(2.9)$ & $3.3(2.9)$ & $5.0(0.0)$ & $5.0(0.0)$ \\
\hline $\begin{array}{r}\text { Distance from front mid-point }(\mathrm{cm}) \\
\text { Rectangle }\end{array}$ & Square & Trapezoid & Semi-circle & Triangle (UP) & Triangle (DS) \\
\hline$-5.3(2.9)$ & $-5.0(0.0)$ & $1.7(2.9)$ & $2.0(0.0)$ & $3.3(2.9)$ & $5.0(0.0)$ \\
\hline $\begin{array}{r}\text { Distance from downstream edge }(\mathrm{cm}) \\
\text { Square }\end{array}$ & Rectangle & Trapezoid & Semi-circle & Triangle (UP) & Triangle (DS) \\
\hline$-15.0(0.0)$ & $-13.3(2.9)$ & $1.7(2.9)$ & $2.0(0.0)$ & $3.3(2.9)$ & $5.0(0.0)$ \\
\hline
\end{tabular}

Table 6. Comparison of means results for obstruction shape versus distance from the upstream edge of the constriction to the riffle crest. Mean values contained within the same horizontal line are not significantly different from each other. Values in parentheses are the corresponding standard deviations.

\begin{tabular}{rrrrrr}
\hline \multicolumn{2}{c}{ Distance from upstream edge (cm) } & & & & \\
Semi-circle & Triangle (UP) & Trapezoid & Triangle (DS) & Rectangle & Square \\
\hline $38.7(2.9)$ & $46.7(5.8)$ & $60.0(5.0)$ & $63.3(20.8)$ & & \\
& $46.7(5.8)$ & $60.0(5.0)$ & $63.3(20.8)$ & $74.3(5.8)$ & \\
& & $60.0(5.0)$ & $63.3(20.8)$ & $74.3(5.8)$ & $78.3(12.6)$ \\
\hline
\end{tabular}

the square and rectangle are positioned furthest downstream. When defined in relation to the upstream edge of the constriction, some statistical minor differences exist (Table 6). The lateral location of the riffle crest was not statistically tested, but the flume-bed topography shows most riffle crests are located near the flume centerline except for the riffle downstream of the triangle UP (Fig. 2). In the later case, the riffle crest appeared to directly build along the left wall of the flume.

The total length of the pool-riffle sequence was significantly related to differences in the obstruction shape. The ANOVA and comparison of means results suggest the lengths of sequences associated with blunt obstructions tend to be similar in characteristics with progressively less overlap in pool-riffle length with the trapezoid, triangle UP and semi circle obstructions. Together, the results suggest the riffle characteristics and resultant pool-riffle length primarily responds to variations in pool scour and subsequent sediment delivery.

\subsection{Backwater development}

The introduction details the role that constrictions to flow play in creating backwater, lateral convergence of flow, jet flow and recirculation in a wake zone downstream of the obstruction. The rectangle, square, triangle DS, and to a lesser degree, the semi-circle shapes created scour even upstream of any change in flume width. The initiation of scour immediately upstream of the obstructions suggests accelerated near-bed velocities with increased bed shear stress adjacent to the constriction (Fig. 2). The most likely cause for the accelerated near-bed velocities would be locally-increased, water-surface slopes associated with backwater development on the obstruction-side relative to the mid-channel section of the flume. In a roughly rectangular-shaped natural constriction in Colorado, Thompson et al. (1999) noted dramatically increased water-surface profiles that tended to wrap around towards the obstruction-side bank at progressively higher flows. The water-surface slopes immediately adjacent to the constriction were the highest anywhere in the pool-riffle sequence, while slopes along the channel centerline tended to be more similar in the pool and riffle (Thompson et al., 1999). In the flume, high water surface slopes upstream of blunt 
obstructions to flow would lead to high bed shear stresses even upstream of the physical constriction.

Obstructions that present a less drastic transition to constricted flow appear to generate less backwater and differences in convergence and jet flow. The triangle UP and the trapezoid-shaped obstructions produced much less scour upstream of the maximum point of constriction, and almost no scour upstream of the obstruction itself (Fig. 2). In these cases, backwater development and water-surface slopes were most likely depressed relative to the blunt obstructions.

\subsection{Flow convergence and vena-contracta formation}

In all six configurations, the deepest portion of the pool formed at least slightly downstream of the initial point of maximum constriction (Fig. 2). As described below, only the semi-circle shape displayed a tendency for upstreamdownstream symmetry of the scour hole around the obstruction. The statistical results for the location of maximum scour along the flume centerline confirm that pool location is strongly related to the position of the upstream edge of the maximum width of constriction (Table 4). In particular, pool locations are statistically insignificant when measured from this point of the constriction. This lack of variation in pool location shows that the convergence of flow past the initial point of minimum width is a primary control on pool characteristics. Even when measured from the initial point of maximum constriction, all the pools display positive mean values for pool location, which indicates slight downstream positioning of the point of maximum scour (Table 5). Thompson (2006) also found the deepest portion of scour holes occurred downstream of the physical obstruction when recirculating eddies influenced pool scour relative to pools without wake zones downstream of a physical constriction.

The pattern of scour highlights the role that continued flow convergence and flow recirculation plays in the formation of a vena contracta. This scour pattern is perhaps best displayed in the triangle DS. The angled scour hole outlines the approximate position of the jet responsible for excavation of the pool. The deepest portion of the pool and widest section of the pool center are located approximately $10 \mathrm{~cm}$ downstream and $5 \mathrm{~cm}$ towards the opposite bank of the point of maximum constriction. The pattern of pool scour displays the continued flow convergence characterized by the formation of a recirculating eddy and vena contracta. The location of maximum scour is similar for the square and rectangle, which may indicate narrow zones of recirculation formed along the outer edge of the constrictions.

In contrast to the other shapes, the semi-circle obstruction produced an interesting curved pool that was almost symmetrical with regard to upstream and downstream depths and positions of scour. The triangle UP produced a somewhat similar shape of the pool, but the scour was still much more developed downstream of the obstruction. Interestingly, the semi-circle-shaped obstruction was the only one that utilized a gradual increase in width with minor increases in angle of the flume wall in relation to the primary flow direction. Therefore, separation of the boundary layer from the obstruction wall would likely be delayed with this shape. The curved nature of the scour hole may indicate this delayed separation of the boundary layer from the obstruction.

Although previous research shows that turbulent scour probably played an important role in increasing the depth of scour downstream of the pools (Thompson, 2006), it was not possible to determine the position of the eddy fence with the associated vortex shedding in any of the flume runs. Therefore, it is unclear what role turbulent scour along shear layers may play in influencing the exact location of the pool center. However, the curved zone of pool scour downstream of the triangle UP, the triangle DS and the semi-circle are possibly a result of vortex development and movement along the outer edge of small recirculation zones downstream of the obstructions. Furthermore, the enhanced scour with different-shaped obstructions indicates that from drag dominated pool development. Despite increased skin friction with longer longitudinal outer surfaces along the square and rectangular obstructions, the pools were still deeper and more voluminous than with the triangular obstructions to flow. These results are consistent with the earlier experiments by Thompson (2006) with long streamlined obstructions versus shorter obstructions with lateral wakes. However, the development of the bed morphology would counteract this trend to some degree as noted by Carling (1989).

The topography of the riffle with the riffle crest often centrally located within the flume (Fig. 2), should enhance lateral flow divergence downstream of the pool. Previous research shows that the combination of strong lateral divergence of flow and an uphill climb for sediment traveling up the pool-exit slope out of the pool should enhance sediment deposition in the region of the riffle (Thompson et al., 1999). It is interesting to note that riffles tended to grow in extent laterally as much as they lengthened with larger supplies of sediment from deeper pools. This trend would tend to further enhance flow divergence with a feedback of enhanced sediment deposition.

\section{Conclusions}

In a forced-pool-riffle sequence, changes in obstruction shape can alter the depth of the pool, the location of the riffle, and the distance between the pool and riffle. During the course of the experiments it appeared that the most important aspect of obstruction shape on pool-riffle characteristics was the point of initial constriction of the water's flow for constrictions with abrupt versus more gradual channel narrowing displaying different responses. Abrupt decreases in channel width presented by obstructions with blunt upstream faces create more backwater development, which leads to deeper, larger pools. The enhanced scour with the blunt 
obstructions produces larger riffles, primarily through deposition of a wider areal extent, uniformed-height bar. The position of the upstream edge of the constriction also influences the downstream distance to the pool center and creates longer pool-riffle-sequence lengths. These combined characteristics have the chance of influencing the basic morphology of the pool-riffle sequence.

Acknowledgements. Paul Carling, Tom Lisle and Bruce MacVicar contributed valuable comments on a draft version of the article. Bailey Fidler provided important help with data collection.

\section{Edited by: M. Gooseff}

\section{References}

Buffington, J. M., Lisle, T. E., Woodsmith, R. D., and Hilton, S.: Controls on the size and occurrence of pools in coarse-grained forest rivers, River Res. App., 18, 507-531, 2002.

Carling, P. A.: Hydrodynamic models of boulder berm deposition, Geomorphology, 2, 319-340, 1989.

Clifford, N. J.: Differential bed sedimentology and the maintenance of a riffle-pool sequence, Catena, 20, 447-468, 1993.

De Serres, B., Roy, A. G., Biron, P. M., and Best, J. L.: Threedimensional structure of flow at a confluence of river channels with discordant beds, Geomorphology, 26, 313-335, 1999.

Dolan, R., Howard, A., and Trimble D.: Structural control of the rapids and pools of the Colorado River in the Grand Canyon, Science, 202, 629-631, 1978.

Harrison, L. R. and Keller, E. A.: Modeling forced pool-riffle hydraulics in a boulder-bed stream, southern California, Geomorphology, 83, 232-248, 2007.

Hassan, M. A. and Woodsmith, R. D.: Bedload transport in an obstructed-formed pool in a forested gravel-bed stream, Geomorphology, 58, 203-221, 2004.

Jia, Y., Kitamura, T., and Wang, S. S.: Simulation of scour process in plunging pool of loose bed-material, J. Hydraul. Eng., 127, 219-229, 2001.

Kieffer, S. W.: The 1983 hydraulic jump in Crystal Rapid: Implications for river-running and geomorphic evolution in the Grand Canyon, J. Geol., 93, 385-406, 1985.

Lisle, T. E.: Stabilization of a gravel channel by large streamside obstructions and bedrock bends, Jacoby Creek, northwest California, Geol. Soc. Am. Bull., 97, 999-1011, 1986.

Lisle, T. E. and Hilton, S.: The volume of fine sediment in pools: an index of sediment supply in gravel-bed streams, Water Resour. Bull., 28, 371-383, 1992.

Liu, P. Q., Dong, J. R., and Yu, C.: Experimental investigation of fluctuation uplift on rock blocks at the bottom of the scour pool downstream of Three-Gorges spillway, J. Hydraul. Res., 3, 5568, 1998.

MacVicar, B. J. and Roy, A. G.: Hydrodynamics of a forced riffle pool in a gravel bed river: 1. Mean velocity and turbulence intensity, Water Resour. Res., 43, W12401, doi:10.1029/2006WR005272, 2007a.

MacVicar, B. J. and Roy, A. G.: Hydrodynamics of a forced riffle pool in a gravel bed river: 2. Scale and structure of coherent turbulent events, Water Resour. Res., 43, W12402, doi:10.1029/2006WR005274, 2007b.
McLean, S. R., Nelson, J. M., and Wolfe, S. R.: Turbulence structure over two-dimensional bed forms: Implications for sediment transport, J. Geophys. Res., 99, 12729-12747, 1994.

MacWilliams Jr., M. L., Wheaton, J. M., Pasternack, G. B., Street, R. L., and Kitanidis, P. K.: Flow convergence routing hypothesis for pool-riffle maintenance in alluvial rivers, Water Resour. Res., 42, W10427, doi:10.1029/2005WR004391, 2006.

Milan, D. J., Heritage, G. L., Large, A. R. G., and Charlton, M. E.: Stage dependent variability in tractive force distribution through a riffle pool sequence, Catena, 44, 85-109, 2001.

Miller, A. J.: Debris-fan constrictions and flood hydraulics in river canyons: Some implications from two-dimensional flow modeling, Earth Surf. Process. Landforms, 19, 681-697, 1994.

Montgomery, D. R., Buffington, J. M., Smith, R. D., Schmidt, K. M., and Pess, G.: Pool spacing in forest channels, Water Resour. Res., 31, 1097-1105, 1995.

Nelson, J. M., McLean, S. R., and Wolfe, S. R.: Mean flow and turbulence fields over two-dimensional bed forms, Water Resour Res., 29, 3935-3953, 1993.

Petit, F.: The relationship between shear stress and the shaping of the bed of a pebble-loaded river, La Rulles-Ardenne, Catena, 14, 453-468, 1987.

Roy, A. G., Biron, P. M., Buffin-Bélanger, T., and Levasseur, M.: Combined visual and quantitative techniques in the study of natural turbulent flows, Water Resour. Res., 35, 871-877, 1999.

Schmidt, J. C., Rubin, D. M., and Ikeda, H.: Flume simulation of recirculating flow and sedimentation, Water Resour. Res., 29, 2925-2939, 1993.

Thompson, D. M.: Random controls on semi-rhythmic spacing of pools and riffles in constriction-dominated rivers, Earth Surf. Process. Landforms, 26, 1195-1212, 2001.

Thompson, D. M.: Channel-bed scour with high versus low deflectors. American Society of Civil Engineers, J. Hydraul. Eng., 128, 640-643, 2002.

Thompson, D. M.: The influence of pool length on local turbulence production and energy slope: A flume experiment, Earth Surf. Process. Landforms, 29, 1341-1358, 2004.

Thompson, D. M.: The role of vortex shedding in the scour of pools, Adv. Water Resourc., 29, 121-129, 2006.

Thompson, D. M.: Turbulence characteristics in a shear zone downstream of a channel constriction in a coarse-grained pool, Geomorphology, 83, 199-214, 2007.

Thompson, D. M. and Hoffman, K. S.: Equilibrium pool dimensions and sediment-sorting patterns in coarse-grained, New England channels, Geomorphology, 38, 301-316, 2001.

Thompson, D. M., Nelson, J. M., and Wohl, E. E.: Interactions between pool geometry and hydraulics, Water Resour. Res., 34, 3673-3681, 1998.

Thompson, D. M., Wohl, E. E., and Jarrett, R. D.: Pool sediment sorting processes and the velocity-reversal hypothesis, Geomorphology, 17, 142-156, 1999.

Wohl, E. E. and Thompson, D. M.: Velocity fluctuations along a small step-pool channel, Earth Surf. Process. Landforms, 25, 353-367, 2000.

Wohl, E. E. and Legleiter, C.: Controls on pool characteristics along a resistant-boundary channel, J. Geol., 111, 103-114, 2003. 